\title{
Analytical study of a cylindrical linear electromagnetic pulsing motor for electric vehicles
}

\begin{abstract}
The cylindrical linear electromagnetic pulsing motor (EMPM) is an alternative electric vehicle (EV) to be simulated in this study. The proposed design on the cylindrical linear EMPM will replace the piston engine in an internal combustion engine (ICE) which produces linear motion. It can eliminate problems related to internal combustion engines (ICE) such as engine weight and friction where fewer components have been used. In this paper, an analytical model was constructed and predicted the magnetic equivalent circuit (MEC) that can solve with the same technique as the electrical circuit. The initial magneto-statics analysis was conducted through the finite element magnetic software (FEMs) for magnetic filed problem so that the magnetic flux relationship could be predicted. Furthermore, the FE modelling and analysis is followed by a MATLAB/Simulink software calculation to predict the cylinder linear EMPM. Finally, the simulation results of the FE models regarding plunger force, thrust, plunger distance, speed, and power motor were presented and compared with the regulated counterparts obtained from the experimental setup.
\end{abstract}

Keyword: Cylinder linear EMPM; ICE; MEC; FEMs; FE modelling 